\title{
Neurolymphomatosis of the Sciatic and Tibial Nerves
}

\author{
Muhammad Talha Yaseen, Pir Abdul Ahad Aziz, Kashif Siddique and Taimoor Ali Tariq \\ Department of Radiology, Shaukat Khanum Memorial Cancer Hospital, Lahore, Pakistan
}

\begin{abstract}
Neurolymphomatosis (NL) is an uncommon clinical condition, characterised by lymphomatous infiltration of the central and/or peripheral nervous system. Most often it is caused by B-cell non-Hodgkin's lymphoma (NHL). Clinically, patients usually present with neuropathy involving the nerve roots, plexuses, peripheral or cranial nerves. NL usually occurs as a complication of prior lymphoma, but it can also present in the form of relapsed lymphoma. It is important to diagnose and start early treatment in all cases of nodal or visceral (including neural) lymphoma with chemo and/or radiation therapy. The PET-CT and MRI can help in making diagnosis. We are presenting a case of 28-year male patient, diagnosed as diffuse large B-cell lymphoma on the background of follicular lymphoma, which initially responded to treatment but then presented with NL, based on clinical history and radiological findings which were confirmed by histopathology.
\end{abstract}

Key Words: Neurolymphomatosis, B-cell non-Hodgkin's lymphoma (NHL), PET.

How to cite this article: Yaseen MT, Aziz PAA, Siddique K, Tariq TA. Neurolymphomatosis of the sciatic and tibial nerves. $J$ Coll Physicians Surg Pak 2019; 29 (Supplement 2):S86-S88.

\section{INTRODUCTION}

Neurolymphomatosis (NL) is a rare clinical complication, which is characterised by nerve infiltration by neurotropic malignant cells in acute leukemia or nonHodgkin's lymphoma (NHL) ${ }^{1}$ with an estimated incidence of $0.2 \% .^{2}$ Magnetic resonance imaging (MRI) and positron emission tomography-computed tomography (PET-CT) are the key imaging tools in making diagnosis. ${ }^{3,4}$

Its early diagnosis and treatment are imperative for better outcome. Herein, we present a case of a 28-year male with diffuse large B-cell lymphoma, who presented with this complication. He responded well to repeat therapy of lymphoma and is doing well till last follow-up.

\section{CASE REPORT}

A 28-year male patient presented to the walk-in clinic in April 2018 with left sided abdominal pain. CT scan was performed which showed large confluent nodal mass in mesentery with diffuse small bowel wall thickening. Patient underwent excisional biopsy of the mesenteric node in the peripheral hospital, which revealed large B-cell lymphoma in the background of follicular lymphoma. Subsequently, patient underwent chemotherapy, to which he responded well. On the follow-up sequential CT scans, there was good partial treatment response (according to Recist criteria); but unfortunately, after few months in February 2019, he presented to ER for right calf pain, swelling and numbness. Subsequently,

Correspondence to: Dr. Pir Abdul Ahad Aziz, Department of

Radiology, Shaukat Khanum Memorial Cancer Hospital,

Lahore, Pakistan

E-mail: abdulahad.q@gmail.com

Received: May 03, 2019; Revised: July 08, 2019;

Accepted: July 20, 2019 ultrasound was requested by ER physician to rule out deep venous thrombosis (DVT). On ultrasound, there was no evidence of DVT. However, diffuse abnormal thickening of the right sciatic nerve was found from midposterior thigh extending down to the popliteal fossa with further thickening of the right tibial and common peroneal nerves (Figure 1 $A, B$ ). For further assessment of ultrasound findings, MRI was requested, which revealed diffuse thickening of the sciatic nerve extending from mid-thigh down to the popliteal region, showing diffuse post-contrast enhancement and diffusion restriction. Similar signal abnormality was also seen involving the tibial nerve in the popliteal fossa with significant signal changes in the associated posterior compartment muscles (Figure $2 \mathrm{~A}-\mathrm{E}$ ). On the basis of these findings, diagnosis of NL, involving the sciatic and tibial nerves, was made. The case was then discussed in lymphoma conference; which further advised PET-CT scan for restaging of lymphoma and assessment of sciatic and tibial nerves and biopsy for confirmatory tissue diagnosis. 18F FDG PET-CT was performed which also showed diffuse hypermetabolic thickening of sciatic and tibial nerves with activity of 21.5 standard uptake value (SUV) and hypodense hypermetabolic posterior compartment muscles (Figure 3A). This further supported the radiological findings of NL. Patient later underwent biopsy of the enlarged hypermetabolic popliteal node, which showed large B-cell lymphoma as direct biopsy from the nerve was refused by the patient. Currently, patient is under chemotherapy and interim scan showed excellent treatment response with significant interval reduction in sciatic and tibial nerves involvement and perineural fat and muscular involvement. However, residual disease still showed mild hypermetabolic activity that is above background liver activity (Figure 3B). 


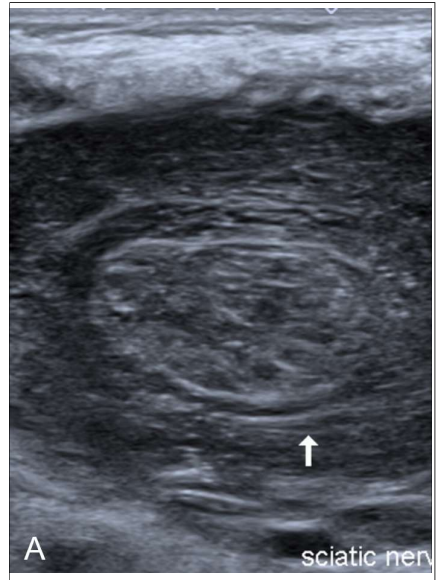

Figure 1A: Ultrasound image (distal right thigh) demonstrating diffuse mass like thickening of the sciatic nerve.

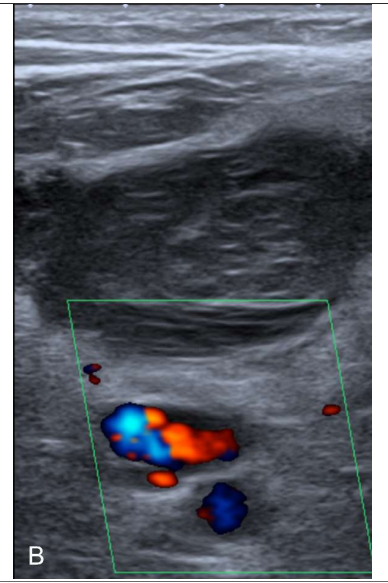

Figure 1B: Ultrasound image (at popliteal fossa) showing diffuse mass like thickening of the sciatic nerve which is displacing the popliteal vessels and causing its compression.

\section{DISCUSSION}

Neurolymphomatosis (NL) is a rare entity; characterised by lymphocytic infiltration of the cranial and peripheral nerves. Literature review shows that $\mathrm{NL}$ is usually seen in relation with $\mathrm{NHL}^{4,5}$ which is in line with our case, in which patient was diagnosed with large B-cell lymphoma on the background of follicular lymphoma on mesenteric nodal biopsy. NL can present as a primary disease or as relapse or progression of a prior treated disease.1,2

Patients with NL usually present with variable symptoms ranging from progressive painful neuropathy involving one or more cranial/peripheral nerves, painful or painless cranial nerve palsies or foot drop.2,6 These patients are treated with systemic chemotherapy, intrathecal chemotherapy and/or radiotherapy. Despite various treatment options, there is poor prognosis for patients with $\mathrm{NL}$ and the disease usually follows a rapidly progressive course.1,3,4 In order to diagnose NL, Doppler ultrasound can be used as initial diagnostic tool as it is non-invasive, readily available and without any radiation exposure. In the cases of NL; ultrasound demonstrates thickening of the involved nerve with increased vascularity on color Doppler images. ${ }^{7} \mathrm{MRI}$ and PET-CT play a key role in the diagnosis of NL with sensitivity of $87.5 \%$ and $100 \%$, respectively. $1,3,8 \mathrm{It}$ is very important to differentiate NL from non-cancerous conditions like inflammatory radiculopathy, Miller Fischer variant of Guillain Barre Syndrome, chemo-radiotherapy induced damage to the peripheral nervous system (PNS), paraneoplastic involvement of the PNS and neurofibromatosis. History is, therefore, very important in such cases to make the definitive diagnosis. Although, histopathology remains the gold standard in the diagnosis of $\mathrm{NL}$ but most patients are usually diagnosed on the basis of clinical history and imaging alone. It is also worthy to mention here that biopsy is usually performed only in

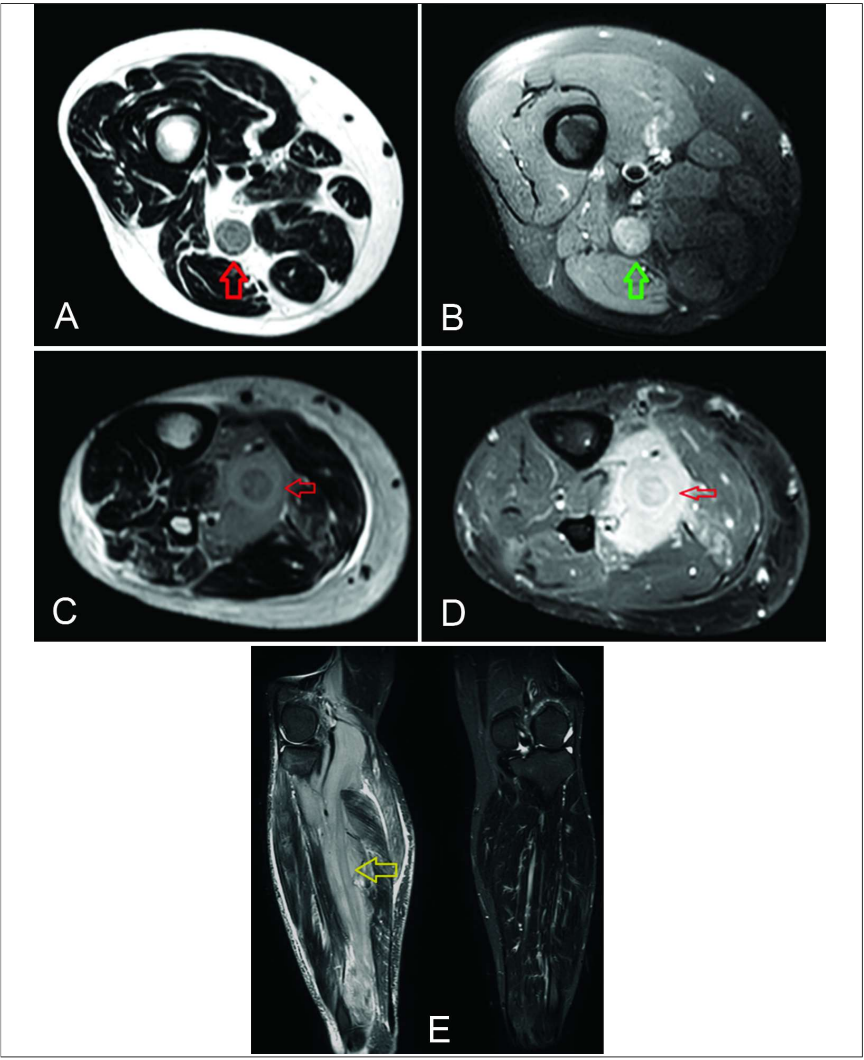

Figure 2: $(A, B) M R I$ axial view T2WI and T1 post-contrast images of right thigh showing diffuse thickening and post-contrast enhancement of the sciatic nerve (red and green arrows). (C,D) Axial view T2WI and T1 postcontrast images of proximal right leg showing diffuse thickening of the tibial nerve with significant perineural signal post-contrast enhancement in adjacent fat and posterior compartment muscles (red arrows). (E) Coronal view STIR images of right leg showing diffuse thickening of the tibial nerve with significant perineural signal post-contrast enhancement in adjacent fat and posterior compartment muscles (yellow arrow).

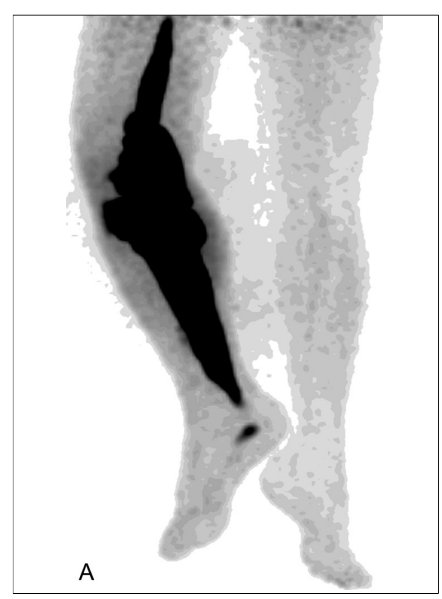

Figure 3A: $18 \mathrm{~F}$ FDG MIP image showing hypermetabolic activity involving the posterior aspect of right mid thigh and along the almost whole length of right leg which corresponded to diffusely thick hypermetabolic sciatic and tibial nerves on CT and PET-CT fusion images (not shown). The maximum SUV of sciatic nerve was 21.5 SUV. Also note hyper-metabolic activity in the calcaneum of 6.6 SUV which did not had any CT correlate; this could be due to focal marrow involvement.

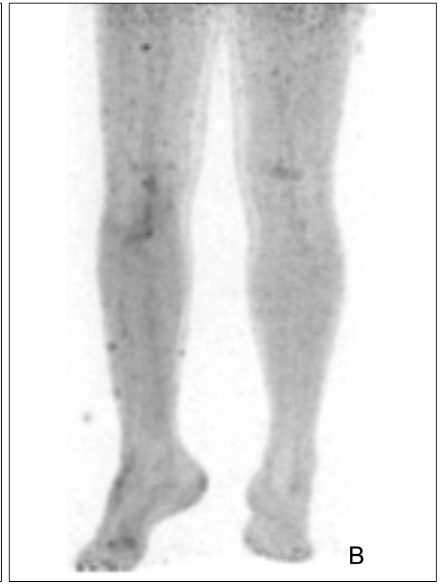

Figure 3B: $18 \mathrm{~F}$ FDG MIP image showing significant interval reduction in previously seen hypermetabolic activity involving the sciatic and tibial nerves in the posterior aspect of right mid thigh and along the almost whole length of right leg, respectively. The maximum SUV of sciatic nerve on current PET scan was noted to be 4.5 SUV (still above background liver $=3.0$ SUV). Further, there is also interval resolution of hypermetabolic deposit in the calcaneum. 
cases in which the diagnosis cannot be made on the basis of clinical history and imaging because of increased risk of permanent nerve damage.9,10

To conclude, NL should be in the differential diagnosis of any neuropathy, especially one that is painful and rapidly evolving in appropriate clinical setting. Radiological investigation plays a vital role, especially in cases where biopsy is difficult to perform or to avoid painful procedure.

\section{PATIENT'S CONSENT:}

The study was given exemption from patient's written/ informed consent by institutional Ethical Review Board.

\section{CONFLICT OF INTEREST:}

Authors declared no conflict of interest.

\section{AUTHORS' CONTRIBUTION:}

MTY: Collected images and case details.

PAAA: Wrote the whole manuscript and participated in data and images collection.

KS: Supervised the whole project.

TAT: Collected the pathological findings and follow-up of patient.

\section{REFERENCES}

1. Choi YJ, Shin JA, Kim YH, Cha SJ, Cho JY, Kang SH, et al. Neurolymphomatosis of brachial plexus in patients with non-
Hodgkin's lymphoma. Case Rep Oncol Med 2013; 2013:492329.

2. Baehring JM, Damek D, Martin EC, Betensky RA, Hochberg FH. Neurolymphomatosis. Neuro Oncol 2003; 5:104-15.

3. Baehring JM, Batchelor TT. Diagnosis and management of neurolymphomatosis. Cancer J 2012; 18:463-8.

4. Grisariu S, Avni B, Batchelor TT, van den Bent MJ, Bokstein F, Schiff D, et al. Neurolymphomatosis: An International Primary CNS Lymphoma Collaborative Group report. Blood 2010; 115: 5005-11.

5. Lim AT, Clucas D, Khoo C, Parameswaran BK, Lau E. Neurolymphomatosis: MRI and (18) FDG-PET features. J Med Imaging Radiat Oncol 2016; 60:92-5.

6. Gan HK, Azad A, Cher L, Mitchell PL. Neurolymphomatosis: Diagnosis, management, and outcomes in patients treated with rituximab. Neuro Oncol 2010; 12:212-5.

7. Vijayan J, Chan YC, Therimadasamy A, Wilder-Smith EP. Role of combined B-mode and Doppler sonography in evaluating neurolymphomatosis. Neurology 2015; 85:752-5.

8. Byun WM, Park WK, Park BH, Ahn SH, Hwang MS, Chang JC. Guillain-Barré syndrome: MR imaging findings of the spine in eight patients. Radiology 1998; 208:137-41.

9. Shree R, Goyal MK, Modi M, Gaspar BL, Radotra BD, Ahuja CK, et al. The diagnostic dilemma of neurolymphomatosis. J Clin Neurol 2016; 12:274-81.

10. Tai R, Maingard J, Nambiar M, Lim K. High-grade B-cell lymphoma relapse presenting as neurolymphomatosis of the median nerve. BMJ Case Rep 2019; 12: pii:e228742. 